Dhaka Univ. J. Biol. Sci. 20(1): 81-89, 2011 (January)

\title{
NUTRIENT UPTAKE, HARVEST AND ADDITION BY CROPS UNDER DIFFERENT CROPPING SEQUENCES
}

\author{
Md. Monjurul Alam, Syed Alauddin Ahmad, A.H. Khan ${ }^{1 *}$ And Sirajul Hoque ${ }^{1}$ \\ Bangladesh Jute Research Institute, Manik Mia Avenue, Dhaka-1207, Bangladesh \\ Key words: Nutrient uptake, Harvest, Addition, Cropping sequence
}

\begin{abstract}
A field trial was conducted to determine the nutrient uptake, harvest and addition by different crops under jute included and jute excluded cropping sequences. The crops were potato (Solanum tuberosum L.), jute (Corchorus olitorius L.), broadcast aus rice and transplanted aman rice (Oryza sativa L.). The soil of the experimental site represents Melandha soil series under noncalcareous brown floodplain soil (General Soil Type) which belongs to Agro Ecological Zone No. 8 (Young Brahmaputra and Jumuna Floodplain). Experiment was conducted with potato as base crop in rabi season for three consecutive years in the same field. The treatments (cropping patterns) were Potato - Jute - T. aman, Potato - B. aus - T. aman, Potato - Fallow - T. aman, and Fallow - Jute - T. aman. Jute plant harvested the lowest amount of nutrients and added back the highest amount to the soil. Contributions to the additions of nutrients by crops followed the following sequence: for nitrogen jute $>$ B. aus $>$ T. aman $>$ potato; for phosphorus jute $>$ T. aman $>$ B. aus $>$ potato; for potassium jute $>$ B. aus $>$ T.aman $>$ potato, and for sulphur jute $>$ B. aus $>$ T. aman $>$ potato.
\end{abstract}

\section{Introduction}

Bangladesh is endowed with a climate favorable for the cultivation of a wide variety of both tropical and temperate crops. Though nearly 100 different kinds of crops are presently grown in Bangladesh, rice the principal one, grows in all the three cropgrowing seasons of the year. Other important crops are wheat, jute, potato, oilseeds, pulses, tobacco, cotton, sugarcane and vegetables. ${ }^{(1)}$

A cropping pattern is a spatial and temporal arrangement of the crops within a cropping year and is largely determined by physical, biological and socio-economic factors. Major cropping patterns used in Bangladesh are: (i) Aus rice/Jute - Fallow - Rabi crops, (ii) Aus rice/Jute - Aman rice - Fallow, (iii) Aus rice/Jute - Aman rice - Rabi crops, (iv) Fallow - Aman rice - Fallow, (v) Mixed rice - Rabi crops, (vi) Rice (B. aman) - Fallow, (vii) Rice (Boro) - Fallow, (viii) Rice (Boro) - Rice (T. aman), and (ix) Mixed aus rice and other kharif crops. A good number of jute included cropping patterns are practiced in different agro-ecological zones of Bangladesh.(2-3) Among those, jute - cabbage, jute - T. amam rice - potato, jute - T. aman rice - wheat, and jute - mustard were found profitable at Manikganj.(3)

*Corresponding author. ${ }^{2}$ Department of Soil, Water \& Environment, University of Dhaka, Dhaka-1000, Bangladesh. 
Higher levels of crop-productivity mean removal of large quantities of plant nutrients. Uptake of $\mathrm{N}, \mathrm{P}, \mathrm{K}$, and $\mathrm{S}$ were respectively 108, 18, 102, and $11 \mathrm{~kg} / \mathrm{ha}$ for rice yield of 6 ton/ha; 131, 20, 193, and $14 \mathrm{~kg} /$ ha for potato yield of 30 ton/ha; and 98, 20, 200, and $35 \mathrm{~kg} / \mathrm{ha}$ for jute fiber yield of 3 ton/ha. ${ }^{(4)}$ It was reported that $\mathrm{N}, \mathrm{P}, \mathrm{K}$, and S were removed from the soil, respectively at a rate of $163,29,169$, and $17.4 \mathrm{~kg} / \mathrm{ha}$ in potato - jute - T. aman sequence; and $279,50,292$, and $45, \mathrm{~kg} / \mathrm{ha}$ in wheat - T. aus - T. aman sequence. ${ }^{(5)}$

Jute, one of the important components of existing cropping pattern, is cultivated in an area of 0.44 million hectares in Bangladesh. ${ }^{(6)}$ It produces huge green leaves, which are ultimately added to the soil. As jute is a tap-rooted crop, it can absorb nutrients from a depth of $60 \mathrm{~cm} .{ }^{(7)}$ Moreover, jute plant can add huge amount of roots, which ultimately are decomposed in soil. Jute leaves, roots and its residues may act in the improvement of soil physico-chemical characteristics. There is a possibility of finding differences in nutrient uptake, harvest and addition by different crops under jute-included and excluded patterns. But little information is available in this regard. An attempt has, therefore, been made to know the nutrient uptake, harvest, and addition prototype by different crops under jute-included and excluded cropping sequences.

\section{Materials and Methods}

The experiments were conducted at the central jute research station of Bangladesh Jute Research Institute (BJRI), Manikganj, Bangladesh, during 2003 to 2006. The crops were potato (Solanum tuberosum L., var. Diamont), jute (Corchorus olitorius L., var. OM-1), broadcast aus rice (Oryza sativa L., var. BRRI Dhan-24) and transplanted aman rice (Oryza sativa L., var. BRRI Dhan-33). Different cropping patterns including and/or excluding jute were used as treatments. The soil of the experimental site represents Melandha soil series under noncalcareous brown floodplain general soil type which belongs to Agro Ecological Zone No. 8 (Young Brahmaputra and Jumuna Floodplain). Before setting the experiment, soil samples were collected and analyzed for particle-size analysis; ${ }^{(8)}$ textural classes; ${ }^{(9)}$ soil bulk density and particle density; ${ }^{(10)}$ soil $\mathrm{pH}$, soil organic carbon and total nitrogen;(11) total $\mathrm{P}^{(12)}$ and total sulphur.(13) Total K was measured by Flame Photometer (Gallenkemp).

The experiment, for three consecutive years, had four treatments (cropping sequence), each having four replications. The individual plots were 6 by $6 \mathrm{~m}$ and had $1 \mathrm{~m}$ distance between adjacent plots. The experiment was arranged in split plot design with year as main plot and treatment as sub-plot. The experimental layout was kept unchanged for entire three years of experiment.

The treatments (cropping patterns) were potato - jute - T. aman (T1), potato - B. aus T. aman (T2), potato - fallow - T. aman (T3) and fallow - jute - T. aman (T4). Fertilizers were applied (Table 1) as per Fertilizer Recommendation Guide of Bangladesh Agriculture Research Council.(4) urea, triple supper phosphate (TSP), murate of potash 
(MP) and gypsum were used for nitrogen, phosphorus, potassium and sulphur, respectively.

Table 1. Treatment combinations with applied nutrients.

\begin{tabular}{llllccc}
\hline Trt. & \multicolumn{2}{c}{ Treatment combinations } & \multicolumn{3}{c}{ Applied nutrients (kg/ha) } \\
\cline { 5 - 7 } & Rabi & Kharif-I & Kharif-II & Rabi & Kharif-I & Kharif-II \\
\cline { 5 - 7 } & & & N - P - K - S & N - P - K - S & N - P - K -S \\
\hline T1 & Potato & Jute & T. aman & $160-30-120-20$ & $90-10-30-20$ & $90-20-40-15$ \\
T2 & Potato & B. aus & T. aman & $160-30-120-20$ & $60-20-35-15$ & $90-20-40-15$ \\
T3 & Potato & Fallow & T. aman & $160-30-120-20$ & -- & $90-20-40-15$ \\
T4 & Fallow & Jute & T. aman & -- & $90-10-30-20$ & $90-20-40-15$ \\
\hline
\end{tabular}

Crop establishment and agronomic practices for potato, jute, broadcast aus rice, and transplanted aman rice were performed as per recommendation by BARI, BJRI, and BRRI, respectively. Removals of nutrients (N, P, K, and S) by the different plant parts of individual crop were measured by using dry matter production and nutrient concentration values. Collected data were analyzed by using MSTAT, a software for statistical analyses. ${ }^{(14)}$

\section{Results and Discussion}

Particle size distribution, textural class, bulk density, particle density, $\mathrm{pH}$, organic matter, total $\mathrm{N}, \mathrm{P}, \mathrm{K}$, and $\mathrm{S}$ contents of the soil were measured before setting the experiment and are presented in Table 2. The soil was a sandy loam with about 19 per cent clay, 25 per cent silt and 56 per cent sand. Its bulk density $\left(1.42 \mathrm{~g} / \mathrm{cm}^{3}\right)$ and particle density $\left(2.56 \mathrm{~g} / \mathrm{cm}^{3}\right)$ values were suitable for the growth of crops. The values of other soil parameters (Table 2) were similar to the values obtained by analysis for the cultivable soils. ${ }^{(15)}$

Table 2. Properties of soils before setting the experiments.

\begin{tabular}{lrrlrr}
\hline Parameters & Values & & Parameters & Values \\
\hline Sand & $55.8 \%$ & & $\mathrm{pH}$ & 6.4 \\
Silt & 25.0 “ & & Organic matter & $1.16 \%$ \\
Clay & 19.2 “ & & Total nitrogen & 0.083 “ \\
Textural class & Sandy loam & & Total phosphorus & 0.006 “ \\
Bulk density & $1.42 \mathrm{~g} / \mathrm{cm}^{3}$ & & Total potassium & 0.359 “ \\
Particle density & $2.56 \mathrm{~g} / \mathrm{cm}^{3}$ & & Total sulphur & 0.041 “ \\
& & & &
\end{tabular}

Nutrient uptake by different plant parts of a crop were summed together to get the TNU by that crop. A portion of total nutrient uptake (TNU) was added back to the soil through roots and leaves nutrient added (NA). The rest was harvested from the soil as 
yield $(\mathrm{NH})$. Nutrient uptake by different crops in the present investigation was in good agreement with the reported values. $(4,16)$

The calculated values of total nutrient uptake, the portion added back to soil and amount harvested or removed by different crops are presented in Table 3 for potato, Table 4 for jute, Table 5 for B. aus, and Table 6 for T. aman crop.

Root, stover and tuber were the parts of potato plant for calculating TNU, tuber and stover for NH, and only root for calculating NA. The highest values of $108.62 \mathrm{~kg} \mathrm{~N} / \mathrm{ha}$ (97.81\%), $17.61 \mathrm{~kg} \mathrm{P} / \mathrm{ha}(97.83 \%), 141.65 \mathrm{~kg} \mathrm{~K} / \mathrm{ha}(97.71 \%)$, and $9.73 \mathrm{~kg} \mathrm{~S} / \mathrm{ha}(96.62 \%)$ were harvested, and $2.43 \mathrm{~kg} \mathrm{~N} / \mathrm{ha}(2.19 \%), 0.39 \mathrm{~kg} \mathrm{P} / \mathrm{ha}(2.17 \%), 3.32 \mathrm{~kg} \mathrm{~K} / \mathrm{ha}(2.29 \%)$, and 0.34 $\mathrm{kg} \mathrm{S} / \mathrm{ha}(3.38 \%)$ were added to the soil from the total uptake of $111.05 \mathrm{~kg} \mathrm{~N} / \mathrm{ha}, 18.00 \mathrm{~kg}$ $\mathrm{P} / \mathrm{ha}, 144.97 \mathrm{~kg} \mathrm{~K} / \mathrm{ha}$, and $10.07 \mathrm{~kg} \mathrm{~S} / \mathrm{ha}$, respectively, by potato crop in T1 of 3rd year of experiment (Table 3 ).

Root, leaf-1 (defoliated during growing period), leaf-2 (with plant during harvest), bark and stick were the parts of jute plant for calculating TNU, bark, stick and leaf-2 for $\mathrm{NH}$, and root and leaf- 1 were taken into account for NA. The highest $79.90 \mathrm{~kg} \mathrm{~N} / \mathrm{ha}$ (36.78\%), $24.58 \mathrm{~kg} \mathrm{P} / \mathrm{ha}(51.09 \%), 142.32 \mathrm{~kg} \mathrm{~K} / \mathrm{ha}(52.50 \%)$, and $16.98 \mathrm{~kg} \mathrm{~S} / \mathrm{ha}(57.40 \%)$ were harvested, and $137.32 \mathrm{~kg} \mathrm{~N} / \mathrm{ha}(63.22 \%), 23.53 \mathrm{~kg} \mathrm{P} / \mathrm{ha}(48.91 \%), 128.74 \mathrm{~kg} \mathrm{~K} / \mathrm{ha}(47.50 \%)$, and $12.60 \mathrm{~kg} \mathrm{~S} / \mathrm{ha}(42.60 \%)$ were added to the soil from the total uptake of $217.22 \mathrm{~kg}$ $\mathrm{N} / \mathrm{ha}, 48.11 \mathrm{~kg} \mathrm{P} / \mathrm{ha}, 271.06 \mathrm{~kg} \mathrm{~K} / \mathrm{ha}$, and $29.58 \mathrm{~kg} \mathrm{~S} / \mathrm{ha}$, respectively, by jute crop in T1 of 3rd year (Table 4 ).

Root, grain and straw were the parts of $\mathrm{B}$. aus rice plant for calculating TNU, grain and straw for NH, and only root for calculating NA. The highest $58.62 \mathrm{~kg} \mathrm{~N} / \mathrm{ha}(94.81 \%)$, $11.73 \mathrm{~kg} \mathrm{P} / \mathrm{ha}(94.60 \%), 78.34 \mathrm{~kg} \mathrm{~K} / \mathrm{ha}(90.74 \%)$, and $7.81 \mathrm{~kg} \mathrm{~S} / \mathrm{ha}(90.50 \%)$ were harvested, and $3.21 \mathrm{~kg} \mathrm{~N} / \mathrm{ha}(5.19 \%), 0.67 \mathrm{~kg} \mathrm{P} / \mathrm{ha}(5.40 \%), 7.99 \mathrm{~kg} \mathrm{~K} / \mathrm{ha}(9.26 \%)$, and $0.82 \mathrm{~kg} \mathrm{~S} / \mathrm{ha}$ $(9.50 \%)$ were added to the soil from the total uptake of $61.83 \mathrm{~kg} \mathrm{~N} / \mathrm{ha}, 12.40 \mathrm{~kg} \mathrm{P} / \mathrm{ha}, 65.19$ $\mathrm{kg} \mathrm{K} / \mathrm{ha}$, and $8.63 \mathrm{~kg} \mathrm{~S} / \mathrm{ha}$, respectively, by B. aus crop in 3rd year (Table 5). Broadcast aus rice was grown in T2 (Potato - B. aus - T. aman sequence) only.

Root, grain and straw were the parts of $\mathrm{T}$. aman rice plant for calculating TNU, grain and straw for NH, and only root for calculating NA. The highest $79.36 \mathrm{~kg} \mathrm{~N} / \mathrm{ha}(96.28 \%)$, $17.91 \mathrm{~kg} \mathrm{P} / \mathrm{ha}(96.08 \%), 100.92 \mathrm{~kg} \mathrm{~K} / \mathrm{ha}(92.68 \%)$, and $10.34 \mathrm{~kg} \mathrm{~S} / \mathrm{ha}(92.16 \%)$ were harvested, and $3.07 \mathrm{~kg} \mathrm{~N} / \mathrm{ha}(3.72 \%), 0.73 \mathrm{~kg} \mathrm{P} / \mathrm{ha}(3.92 \%), 7.97 \mathrm{~kg} \mathrm{~K} / \mathrm{ha}(7.32 \%)$, and 0.88 $\mathrm{kg} \mathrm{S} / \mathrm{ha}(7.84 \%)$ were added to the soil from the total uptake of $82.43 \mathrm{~kg} \mathrm{~N} / \mathrm{ha}, 18.64 \mathrm{~kg}$ $\mathrm{P} / \mathrm{ha}, 108.89 \mathrm{~kg} \mathrm{~K} / \mathrm{ha}$, and $11.22 \mathrm{~kg} \mathrm{~S} / \mathrm{ha}$, respectively, by $\mathrm{T}$. aman in T1 of $3^{\text {rd }}$ year of experiment (Table 6).

The data presented in figures reveal that jute performed the best in terms of nutrient addition to the soil. Even though, the total uptake of N (188.01 kg/ha), P (40.64 kg/ha), K $(240.78 \mathrm{~kg} / \mathrm{ha})$, and S $(24.47 \mathrm{~kg} / \mathrm{ha})$ were maximum by jute, it also returned maximum amount of nutrients to the soil. Potato, B. aus and T. aman crops added back a very small 
Table 3. Nutrient uptake, harvested, and added (kg/ha) by potato crop.

\begin{tabular}{|c|c|c|c|c|c|c|c|c|c|}
\hline \multirow{2}{*}{$\begin{array}{l}\text { Treat- } \\
\text { ment }\end{array}$} & \multirow[t]{2}{*}{ Year } & \multirow{2}{*}{$\begin{array}{l}\text { Nut- } \\
\text { rient }\end{array}$} & \multicolumn{4}{|c|}{ Uptake by potato crop } & \multicolumn{2}{|c|}{ Harvested by } & \multirow{2}{*}{$\begin{array}{l}\text { Added } \\
\text { through } \\
\text { Root } \% 1\end{array}$} \\
\hline & & & Root & Tuber & Stover & Total & Tuber + stover & $\%^{1}$ & \\
\hline \multirow[t]{12}{*}{$\mathrm{T} 1$} & $1^{\text {st }}$ & $\mathrm{N}$ & 2.32 & 92.59 & 9.51 & 104.42 & 102.10 & 97.78 & 2.22 \\
\hline & & $\mathrm{P}$ & 0.33 & 14.12 & 1.89 & 16.34 & 16.01 & 97.98 & 2.02 \\
\hline & & $\mathrm{K}$ & 3.20 & 119.70 & 13.42 & 136.32 & 133.12 & 97.65 & 2.35 \\
\hline & & S & 0.28 & 6.21 & 2.27 & 8.76 & 8.48 & 96.80 & 3.20 \\
\hline & $2^{\text {nd }}$ & $\mathrm{N}$ & 2.47 & 96.20 & 10.27 & 108.94 & 106.47 & 97.73 & 2.27 \\
\hline & & $\mathrm{P}$ & 0.39 & 14.67 & 2.08 & 17.14 & 16.75 & 97.72 & 2.28 \\
\hline & & K & 3.32 & 124.95 & 14.43 & 142.70 & 139.38 & 97.67 & 2.33 \\
\hline & & S & 0.31 & 7.04 & 2.55 & 9.90 & 9.59 & 96.87 & 3.13 \\
\hline & $3^{\text {rd }}$ & $\mathrm{N}$ & 2.43 & 97.89 & 10.73 & 111.05 & 108.62 & 97.81 & 2.19 \\
\hline & & $\mathrm{P}$ & 0.39 & 15.52 & 2.09 & 18.00 & 17.61 & 97.83 & 2.17 \\
\hline & & K & 3.32 & 127.14 & 14.51 & 144.97 & 141.65 & 97.71 & 2.29 \\
\hline & & $S$ & 0.34 & 7.16 & 2.57 & 10.07 & 9.73 & 96.62 & 3.38 \\
\hline \multirow[t]{12}{*}{$\mathrm{T} 2$} & $1^{\text {st }}$ & $\mathrm{N}$ & 2.36 & 92.11 & 9.80 & 104.27 & 101.91 & 97.74 & 2.26 \\
\hline & & $\mathrm{P}$ & 0.36 & 14.13 & 1.77 & 16.26 & 15.90 & 97.79 & 2.21 \\
\hline & & K & 3.26 & 119.80 & 13.46 & 136.52 & 133.26 & 97.61 & 2.39 \\
\hline & & $S$ & 0.30 & 6.22 & 2.34 & 8.86 & 8.56 & 96.61 & 3.39 \\
\hline & $2^{\text {nd }}$ & $\mathrm{N}$ & 2.33 & 92.14 & 9.54 & 104.01 & 101.68 & 97.76 & 2.24 \\
\hline & & $\mathrm{P}$ & 0.36 & 13.57 & 1.83 & 15.76 & 15.40 & 97.72 & 2.28 \\
\hline & & K & 3.15 & 119.84 & 13.34 & 136.33 & 133.18 & 97.69 & 2.31 \\
\hline & & S & 0.30 & 5.65 & 2.28 & 8.23 & 7.93 & 96.35 & 3.65 \\
\hline & $3^{\text {rd }}$ & $\mathrm{N}$ & 2.37 & 91.93 & 9.53 & 103.83 & 101.46 & 97.72 & 2.28 \\
\hline & & $\mathrm{P}$ & 0.36 & 13.54 & 1.82 & 15.72 & 15.36 & 97.71 & 2.29 \\
\hline & & $\mathrm{K}$ & 3.26 & 119.57 & 13.17 & 136.00 & 132.74 & 97.60 & 2.40 \\
\hline & & $S$ & 0.30 & 5.64 & 2.20 & 8.14 & 7.84 & 96.31 & 3.69 \\
\hline \multirow[t]{12}{*}{$\mathrm{T} 3$} & $1^{\text {st }}$ & $\mathrm{N}$ & 2.40 & 91.35 & 9.59 & 103.34 & 100.94 & 97.68 & 2.32 \\
\hline & & $\mathrm{P}$ & 0.36 & 14.10 & 1.82 & 16.28 & 15.92 & 97.79 & 2.21 \\
\hline & & K & 3.17 & 119.55 & 13.29 & 136.01 & 132.84 & 97.67 & 2.33 \\
\hline & & $S$ & 0.30 & 6.20 & 2.32 & 8.82 & 8.52 & 96.60 & 3.40 \\
\hline & $2^{\text {nd }}$ & $\mathrm{N}$ & 2.33 & 92.36 & 9.75 & 104.44 & 102.11 & 97.77 & 2.23 \\
\hline & & $\mathrm{P}$ & 0.36 & 13.60 & 1.84 & 15.80 & 15.44 & 97.72 & 2.28 \\
\hline & & $\mathrm{K}$ & 3.21 & 120.12 & 13.29 & 136.62 & 133.41 & 97.65 & 2.35 \\
\hline & & $S$ & 0.30 & 6.23 & 2.28 & 8.81 & 8.51 & 96.59 & 3.41 \\
\hline & $3^{\text {rd }}$ & $\mathrm{N}$ & 2.34 & 92.18 & 9.75 & 104.27 & 101.93 & 97.76 & 2.24 \\
\hline & & $\mathrm{P}$ & 0.36 & 13.57 & 1.90 & 15.83 & 15.47 & 97.73 & 2.27 \\
\hline & & K & 3.27 & 119.89 & 13.29 & 136.45 & 133.18 & 97.60 & 2.40 \\
\hline & & $S$ & 0.30 & 6.22 & 2.28 & 8.80 & 8.50 & 96.59 & 3.41 \\
\hline
\end{tabular}

1Percentage of total uptake. 
Table 4. Nutrient uptake, harvested and added (kg/ha) by jute crop.

\begin{tabular}{|c|c|c|c|c|c|c|c|c|c|c|c|c|}
\hline \multirow{2}{*}{$\begin{array}{l}\text { Treat- } \\
\text { ment }\end{array}$} & \multirow[t]{2}{*}{ Yr } & \multirow{2}{*}{$\begin{array}{l}\text { Nut- } \\
\text { rients }\end{array}$} & \multicolumn{6}{|c|}{$\begin{array}{l}\text { Uptake by } \\
\text { jute crop }\end{array}$} & \multicolumn{2}{|c|}{$\begin{array}{c}\text { Harvested } \\
\text { by }\end{array}$} & \multicolumn{2}{|c|}{$\begin{array}{l}\text { Added } \\
\text { through }\end{array}$} \\
\hline & & & Root & Leaf-1 & Leaf-2 & Bark & Stick & Total & $\begin{array}{l}\text { Leaf-2 } \\
+ \text { Bark } \\
+ \text { Stick } \\
\end{array}$ & $\%^{1}$ & $\begin{array}{l}\text { Root+ } \\
\text { Leaf-1 }\end{array}$ & $\%^{1}$ \\
\hline \multirow[t]{12}{*}{$\mathrm{T} 1$} & $1^{\text {st }}$ & $\mathrm{N}$ & 9.14 & 122.90 & 27.81 & 24.97 & 22.41 & 207.23 & 75.19 & 36.28 & 132.04 & 63.72 \\
\hline & & $\mathrm{P}$ & 5.38 & 16.86 & 3.82 & 12.21 & 6.11 & 44.38 & 22.14 & 49.89 & 22.24 & 50.11 \\
\hline & & K & 33.87 & 89.18 & 20.18 & 84.90 & 29.20 & 257.33 & 134.28 & 52.18 & 123.05 & 47.82 \\
\hline & & $S$ & 3.49 & 8.24 & 1.87 & 6.66 & 6.79 & 27.05 & 15.32 & 56.64 & 11.73 & 43.36 \\
\hline & $2^{\text {nd }}$ & $\mathrm{N}$ & 9.11 & 127.30 & 28.04 & 25.84 & 23.99 & 214.28 & 77.87 & 36.34 & 136.41 & 63.66 \\
\hline & & $\mathrm{P}$ & 5.52 & 17.85 & 3.93 & 13.20 & 6.35 & 46.85 & 23.48 & 50.12 & 23.37 & 49.88 \\
\hline & & K & 34.80 & 92.37 & 20.35 & 88.41 & 30.35 & 266.28 & 139.11 & 52.24 & 127.17 & 47.76 \\
\hline & & $S$ & 3.59 & 8.54 & 1.88 & 6.89 & 7.76 & 28.66 & 16.53 & 57.68 & 12.13 & 42.32 \\
\hline & $3^{\text {rd }}$ & $\mathrm{N}$ & 9.40 & 127.92 & 28.38 & 27.05 & 24.47 & 217.22 & 79.90 & 36.78 & 137.32 & 63.22 \\
\hline & & $\mathrm{P}$ & 5.53 & 18.00 & 3.99 & 14.11 & 6.48 & 48.11 & 24.58 & 51.09 & 23.53 & 48.91 \\
\hline & & K & 34.85 & 93.89 & 20.83 & 90.55 & 30.94 & 271.06 & 142.32 & 52.50 & 128.74 & 47.50 \\
\hline & & $S$ & 3.60 & 9.00 & 2.00 & 7.06 & 7.92 & 29.58 & 16.98 & 57.40 & 12.60 & 42.60 \\
\hline \multirow[t]{12}{*}{$\mathrm{T} 4$} & $1^{\text {st }}$ & $\mathrm{N}$ & 8.41 & 106.06 & 23.91 & 22.11 & 18.98 & 179.47 & 65.00 & 36.22 & 114.47 & 63.78 \\
\hline & & $\mathrm{P}$ & 4.70 & 14.14 & 3.19 & 11.06 & 4.90 & 37.99 & 19.15 & 50.41 & 18.84 & 49.59 \\
\hline & & K & 31.16 & 79.46 & 17.91 & 76.38 & 25.71 & 230.62 & 120.00 & 52.03 & 110.62 & 47.97 \\
\hline & & $S$ & 2.97 & 6.73 & 1.52 & 5.53 & 6.12 & 22.87 & 13.17 & 57.59 & 9.70 & 42.41 \\
\hline & $2^{\text {nd }}$ & $\mathrm{N}$ & 8.54 & 105.91 & 24.05 & 22.19 & 19.19 & 179.88 & 65.43 & 36.37 & 114.45 & 63.63 \\
\hline & & $\mathrm{P}$ & 4.77 & 14.17 & 3.22 & 11.09 & 4.95 & 38.20 & 19.26 & 50.42 & 18.94 & 49.58 \\
\hline & & K & 31.64 & 79.60 & 18.08 & 76.65 & 26.62 & 232.59 & 121.35 & 52.17 & 111.24 & 47.83 \\
\hline & & $S$ & 3.01 & 6.75 & 1.53 & 5.55 & 6.19 & 23.03 & 13.27 & 57.62 & 9.76 & 42.38 \\
\hline & $3^{\text {rd }}$ & $\mathrm{N}$ & 8.63 & 107.11 & 24.81 & 22.68 & 19.57 & 182.80 & 67.06 & 36.68 & 115.74 & 63.32 \\
\hline & & $\mathrm{P}$ & 5.08 & 13.99 & 3.24 & 11.34 & 5.05 & 38.70 & 19.63 & 50.72 & 19.07 & 49.28 \\
\hline & & K & 31.99 & 81.18 & 18.80 & 78.87 & 27.15 & 237.99 & 124.82 & 52.45 & 113.17 & 47.55 \\
\hline & & $S$ & 3.05 & 6.82 & 1.58 & 5.67 & 6.31 & 23.43 & 13.56 & 57.87 & 9.87 & 42.13 \\
\hline
\end{tabular}

${ }^{1}$ Percentage of total uptake.

Table 5. Nutrient uptake, harvested and added (kg/ha) by B. aus crop.

\begin{tabular}{|c|c|c|c|c|c|c|c|c|c|}
\hline \multirow{2}{*}{$\begin{array}{l}\text { Treat- } \\
\text { ment }\end{array}$} & \multirow[t]{2}{*}{ Year } & \multirow{2}{*}{$\begin{array}{l}\text { Nut- } \\
\text { rient }\end{array}$} & \multicolumn{4}{|c|}{ Uptake by B. aus crop } & \multicolumn{2}{|c|}{ Harvested by } & \multirow{2}{*}{$\begin{array}{l}\text { Added through } \\
\text { root } \%^{1}\end{array}$} \\
\hline & & & Root & Grain & Straw & Total & Grain + straw & $\%^{1}$ & \\
\hline \multirow[t]{12}{*}{$\mathrm{T} 2$} & $1^{\text {st }}$ & $\mathrm{N}$ & 3.25 & 33.15 & 25.05 & 61.45 & 58.2 & 94.71 & 5.29 \\
\hline & & $\mathrm{P}$ & 0.60 & 8.22 & 3.47 & 12.29 & 11.69 & 95.12 & 4.88 \\
\hline & & K & 8.00 & 10.08 & 68.58 & 86.66 & 78.66 & 90.77 & 9.23 \\
\hline & & $S$ & 0.83 & 2.92 & 4.62 & 8.37 & 7.54 & 90.08 & 9.92 \\
\hline & $2^{\text {nd }}$ & $\mathrm{N}$ & 3.19 & 33.42 & 24.94 & 61.55 & 58.36 & 94.82 & 5.18 \\
\hline & & $\mathrm{P}$ & 0.66 & 8.22 & 3.45 & 12.33 & 11.67 & 94.65 & 5.35 \\
\hline & & K & 7.88 & 10.08 & 67.53 & 85.49 & 77.61 & 90.78 & 9.22 \\
\hline & & $S$ & 0.82 & 2.92 & 4.60 & 8.34 & 7.52 & 90.17 & 9.83 \\
\hline & $3^{\text {rd }}$ & $\mathrm{N}$ & 3.21 & 33.62 & 25.00 & 61.83 & 58.62 & 94.81 & 5.19 \\
\hline & & $\mathrm{P}$ & 0.67 & 8.27 & 3.46 & 12.40 & 11.73 & 94.60 & 5.40 \\
\hline & & K & 7.99 & 10.67 & 67.67 & 86.33 & 78.34 & 90.74 & 9.26 \\
\hline & & S & 0.82 & 3.20 & 4.61 & 8.63 & 7.81 & 90.50 & 9.50 \\
\hline
\end{tabular}

${ }^{1}$ Percentage of total uptake. 
Table 6. Nutrient uptake, harvested and added (kg/ha) by T. aman crop.

\begin{tabular}{|c|c|c|c|c|c|c|c|c|c|}
\hline \multirow{2}{*}{$\begin{array}{l}\text { Treat- } \\
\text { ment }\end{array}$} & \multirow[t]{2}{*}{ Year } & \multirow{2}{*}{$\begin{array}{l}\text { Nut- } \\
\text { rient }\end{array}$} & \multicolumn{4}{|c|}{ Uptake by T. aman crop } & \multicolumn{2}{|c|}{ Harvested by } & \multirow{2}{*}{$\begin{array}{l}\text { Added through } \\
\text { root } \%^{1}\end{array}$} \\
\hline & & & Root & Grain & Straw & Total & Grain + straw & $\% 1$ & \\
\hline \multirow[t]{12}{*}{$\mathrm{T} 1$} & \multirow[t]{4}{*}{$1^{\text {st }}$} & $\mathrm{N}$ & 2.80 & 43.16 & 31.12 & 77.08 & 74.28 & 96.37 & 3.63 \\
\hline & & $\mathrm{P}$ & 0.62 & 10.70 & 4.18 & 15.50 & 14.88 & 96.00 & 4.00 \\
\hline & & K & 7.32 & 14.16 & 78.95 & 100.43 & 93.11 & 92.71 & 7.29 \\
\hline & & S & 0.28 & 4.14 & 5.11 & 9.53 & 9.25 & 97.06 & 2.94 \\
\hline & \multirow[t]{4}{*}{$2^{\text {nd }}$} & $\mathrm{N}$ & 2.87 & 44.73 & 32.10 & 79.70 & 76.83 & 96.40 & 3.60 \\
\hline & & $\mathrm{P}$ & 0.63 & 11.81 & 4.79 & 17.23 & 16.60 & 96.34 & 3.66 \\
\hline & & K & 7.63 & 15.03 & 82.41 & 105.07 & 97.44 & 92.74 & 7.26 \\
\hline & & S & 0.84 & 4.29 & 5.27 & 10.40 & 9.56 & 91.92 & 8.08 \\
\hline & \multirow[t]{4}{*}{$3^{\text {rd }}$} & $\mathrm{N}$ & 3.07 & 45.75 & 33.61 & 82.43 & 79.36 & 96.28 & 3.72 \\
\hline & & $\mathrm{P}$ & 0.73 & 11.89 & 6.02 & 18.64 & 17.91 & 96.08 & 3.92 \\
\hline & & K & 7.97 & 15.13 & 85.79 & 108.89 & 100.92 & 92.68 & 7.32 \\
\hline & & $S$ & 0.88 & 4.32 & 6.02 & 11.22 & 10.34 & 92.16 & 7.84 \\
\hline \multirow[t]{12}{*}{$\mathrm{T} 2$} & \multirow[t]{4}{*}{$1^{\text {st }}$} & $\mathrm{N}$ & 2.27 & 35.90 & 24.94 & 63.11 & 60.84 & 96.40 & 3.60 \\
\hline & & $\mathrm{P}$ & 0.45 & 8.76 & 3.07 & 12.28 & 11.83 & 96.34 & 3.66 \\
\hline & & K & 5.95 & 11.68 & 64.46 & 82.09 & 76.14 & 92.75 & 7.25 \\
\hline & & S & 0.68 & 2.92 & 3.84 & 7.44 & 6.76 & 90.86 & 9.14 \\
\hline & \multirow[t]{4}{*}{$2^{\text {nd }}$} & $\mathrm{N}$ & 2.36 & 36.78 & 26.24 & 65.38 & 63.02 & 96.39 & 3.61 \\
\hline & & $\mathrm{P}$ & 0.47 & 8.97 & 3.18 & 12.62 & 12.15 & 96.28 & 3.72 \\
\hline & & K & 6.19 & 11.96 & 66.78 & 84.93 & 78.74 & 92.71 & 7.29 \\
\hline & & $S$ & 0.71 & 2.99 & 3.98 & 7.68 & 6.97 & 90.76 & 9.24 \\
\hline & \multirow[t]{4}{*}{$3^{\text {rd }}$} & $\mathrm{N}$ & 2.41 & 37.41 & 26.29 & 66.11 & 63.70 & 96.35 & 3.65 \\
\hline & & $\mathrm{P}$ & 0.47 & 9.05 & 3.59 & 13.11 & 12.64 & 96.41 & 3.59 \\
\hline & & K & 6.23 & 12.37 & 66.53 & 85.13 & 78.90 & 92.68 & 7.32 \\
\hline & & $S$ & 0.65 & 3.02 & 3.98 & 7.65 & 7.00 & 91.50 & 8.50 \\
\hline \multirow[t]{12}{*}{ T3 } & \multirow[t]{4}{*}{$1^{\text {st }}$} & $\mathrm{N}$ & 2.36 & 37.11 & 26.35 & 65.82 & 63.46 & 96.41 & 3.59 \\
\hline & & $P$ & 0.47 & 9.05 & 3.19 & 12.71 & 12.24 & 96.30 & 3.70 \\
\hline & & K & 6.20 & 12.37 & 67.48 & 86.05 & 79.85 & 92.79 & 7.21 \\
\hline & & $S$ & 0.71 & 3.32 & 4.39 & 8.42 & 7.71 & 91.57 & 8.43 \\
\hline & \multirow[t]{4}{*}{$2^{\text {nd }}$} & $\mathrm{N}$ & 2.46 & 39.18 & 27.27 & 68.91 & 66.45 & 96.43 & 3.57 \\
\hline & & $\mathrm{P}$ & 0.49 & 9.48 & 3.72 & 13.69 & 13.20 & 96.42 & 3.58 \\
\hline & & K & 6.51 & 12.96 & 69.83 & 89.30 & 82.79 & 92.71 & 7.29 \\
\hline & & $S$ & 0.68 & 3.48 & 4.13 & 8.29 & 7.61 & 91.80 & 8.20 \\
\hline & \multirow[t]{4}{*}{$3^{\text {rd }}$} & $\mathrm{N}$ & 2.58 & 39.51 & 28.07 & 70.16 & 67.58 & 96.32 & 3.68 \\
\hline & & $\mathrm{P}$ & 0.57 & 9.88 & 3.83 & 14.28 & 13.71 & 96.01 & 3.99 \\
\hline & & K & 6.66 & 13.06 & 71.45 & 91.17 & 84.51 & 92.69 & 7.31 \\
\hline & & S & 0.69 & 3.19 & 4.68 & 8.56 & 7.87 & 91.94 & 8.06 \\
\hline \multirow[t]{12}{*}{$\mathrm{T} 4$} & $1^{\mathrm{rd}}$ & $\mathrm{N}$ & 2.46 & 38.40 & 27.04 & 67.90 & 65.44 & 96.38 & 3.62 \\
\hline & & $\mathrm{P}$ & 0.48 & 9.60 & 3.69 & 13.77 & 13.29 & 96.51 & 3.49 \\
\hline & & K & 6.37 & 12.70 & 69.65 & 88.72 & 82.35 & 92.82 & 7.18 \\
\hline & & $S$ & 0.72 & 3.72 & 4.51 & 8.95 & 8.23 & 91.96 & 8.04 \\
\hline & $2^{\text {nd }}$ & $\mathrm{N}$ & 2.63 & 40.39 & 28.82 & 71.84 & 69.21 & 96.34 & 3.66 \\
\hline & & $\mathrm{P}$ & 0.58 & 10.10 & 4.37 & 15.05 & 14.47 & 96.15 & 3.85 \\
\hline & & K & 6.80 & 13.35 & 74.22 & 94.37 & 87.57 & 92.79 & 7.21 \\
\hline & & $S$ & 0.77 & 3.91 & 4.80 & 9.48 & 8.71 & 91.88 & 8.12 \\
\hline & $3^{\text {rd }}$ & $\mathrm{N}$ & 2.73 & 41.28 & 29.27 & 73.28 & 70.55 & 96.27 & 3.73 \\
\hline & & $\mathrm{P}$ & 0.59 & 10.57 & 4.44 & 15.60 & 15.01 & 96.22 & 3.78 \\
\hline & & K & 7.02 & 13.54 & 75.84 & 96.40 & 89.38 & 92.72 & 7.28 \\
\hline & & $\mathrm{S}$ & 0.78 & 3.96 & 5.32 & 10.06 & 9.28 & 92.25 & 7.75 \\
\hline
\end{tabular}

${ }^{1}$ Percentage of total uptake. 
口TNU $\square$ NH $\square$ NA

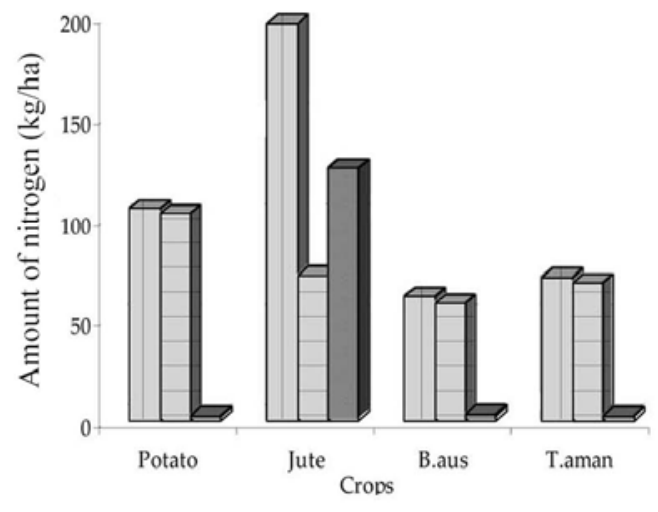

Fig. 1. Amount of nitrogen $(\mathrm{kg} / \mathrm{ha})$ uptake, harvested and added by potato, jute, B. aus, and T. aman crops (average of three years).

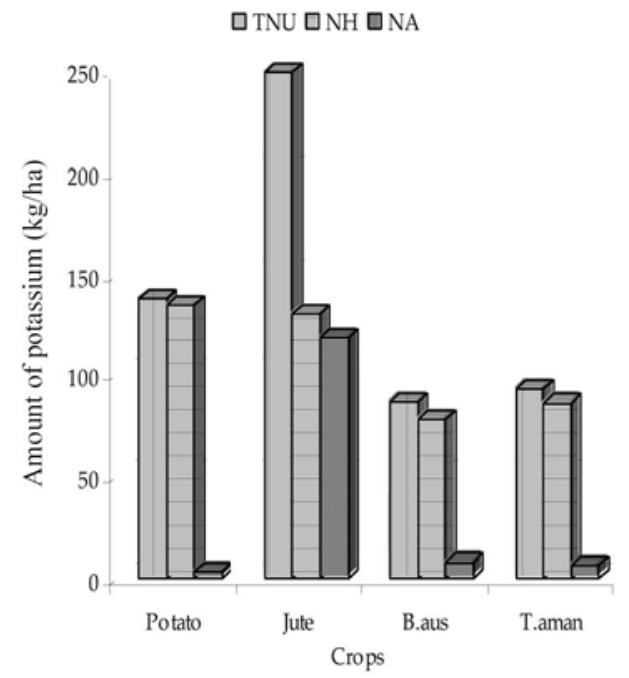

Fig. 3. Amount of potassium (kg/ha) uptake, harvested and added by potato, wheat, jute, B. aus, and T. aman crops (average of three years). $\square T N U \square N H \square N A$

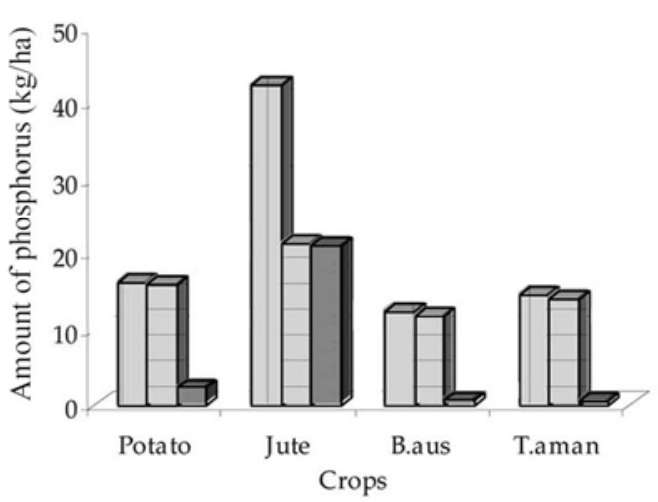

Fig. 2. Amount of phosphorus ( $\mathrm{kg} / \mathrm{ha})$ uptake, harvested and added by potato, jute, B. aus, and T. aman crops (average of three years).

QTNU QNH QNA

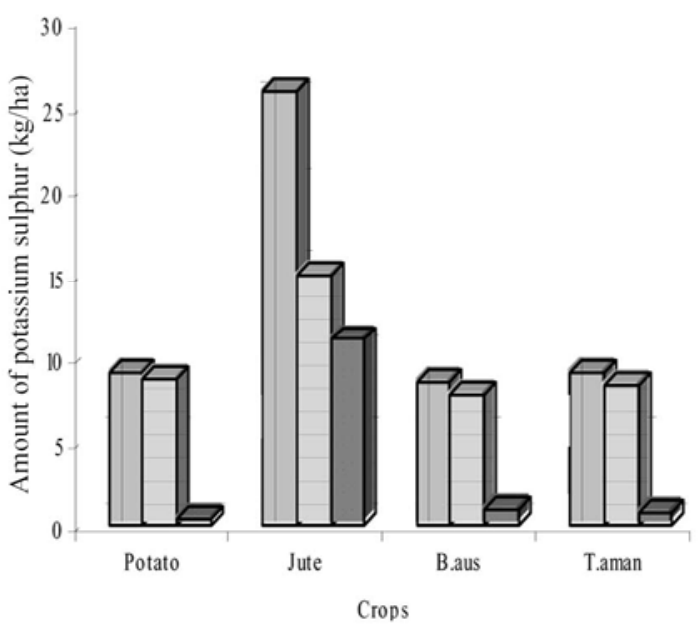

Fig. 4. Amount of sulphur (kg/ha) uptake, harvested and added by potato, wheat, jute, B. aus, and T. aman crops (average of three years).

amount of nutrients to the soil. The average nutrient addition (percentage of total nutrient uptake) by these crops ranged from 2.25 to 5.09 for N, 2.20 to 5.16 for P, 2.38 to 9.25 for $\mathrm{K}$, and 3.36 to 9.72 for $\mathrm{S}$. But in case of jute the nutrient addition (percentage of total nutrient uptake) was 63.74 for $\mathrm{N}, 49.58$ for $\mathrm{P}, 47.91$ for $\mathrm{K}$, and 41.07 for S. From the above observation it is clear that inclusion of jute in kharif-I season helped the following crops in the sequence supplying nutrients to the soil. 


\section{References}

1. Bhuiyan NI 1992. Intensive cropping and soil nutrient balance in Bangladesh. In: M.S. Hussain, S.M.I. Huq, M.A. Iqbal and T.H. Khan (ed.) Improving soil management for intensive cropping in the Tropics and Subtropics. Proceedings of the Inter-Congress Conference of Commission IV. 1-3 Dec. 1992. Dhaka, Bangladesh.

2. Azad AK and MN Nabi 1982. Agro-economic analysis of cropping pattern involving jute and potato. Ban. J. Jute \& Fib. Res. 7(1\&2): 129-139.

3. Azad AK, FAH Talukder, MA Wahab and MA Khan 1984. Progress and prospect of jute based cropping system research in Bangladesh. Expert Consultations, Jute and Kenaf improvement, 12-15 September, Bangladesh Jute Res. Inst. Dhaka. Pp. 240-253.

4. BARC, 2005. Fertilizer recommendation guide, Soils Publication No. 45. Published by Bangladesh Agricultural Research Council, Farmgate, New Airport Road, Dhaka-1215, Bangladesh.

5. Islam MS 1992. Integrated nutrient management approach for fertilizer recommendation in cropping patterns under different soil conditions of Bangladesh. In: M.S. Hussain, S.M.I. Huq, M.A. Iqbal and T.H. Khan (ed.) Improving soil management for intensive cropping in the Tropics and Subtropics. Proceedings of the Inter-Congress Conference of Commission IV. 1-3 Dec. 1992. Dhaka, Bangladesh.

6. BBS 2004. Statistical yearbook of Bangladesh. Bangladesh Bureau of Statistics. Ministry of planning. Govt. of Bangladesh.

7. Ghosh T 1983. Handbook on Jute, Plant Production and Protection, Paper-51, Food and Agriculture Organization, United Nations, Rome, Italy.

8. Day PR 1965. Particle formation and particle-size analysis. In: Methods of soil analysis. C. A. Black, D. D. Evens J. L. White, L. E. Ensuinger and F. E. Clark (ed). Methods of Soil Analysis, Part-I. Agron. Monog, Ame. Soc. Agron. Academic Press, New York. pp. 545567.

9. Marshall CE 1951. Proportion of sand, silt and clay in the basic soil textural classes. U.S. Dept. Agric. Handbook No. 18, Soil Survey Manual, USA. p. 503.

10. Blake CA 1965. Methods of soil analysis. Part-I. Am. Soc. Agron., Madison, Wisconsin, USA.

11. Jackson ML 1973. Soil chemical analysis. Prentice Hall of India, (Pvt.) Ltd. New Delhi, India.

12. Murphy J and JP Riley 1962. A modified single solution method for the determination of phosphate in natural waters. Anal. Chem. Acta. 27: 31-36.

13. Hunt, 1980. Determination of total sulphur in small amount of plant material. Analyst 105: 8385.

14. MSTAT 2002. Design, Management, and Statistical Research Tool, Version 1.4. Plant and Soil Sciences, Michigan State University, East Lansing, MI 48824, USA.

15. Alam M Monjurul, AKM Maqsudul Alam and S Haque 2002. Fertilizer Management of Late Jute Seed Production in Different Agro-Ecological Zones of Bangladesh. Pakistan J. Biol. Sci. 5(4): 410-412.

16. Singh SS 2003. Soil Fertility and Nutrient Management. Kalyani Publishrs, New Delhi-110 002, India. 